III. IEUTRON STAR FORMATION IN THEORETICAL SUPERNOVAE

CHAIR: R. Chevalier 


\title{
THE BIRTH OF NEUTRON STARS
}

\author{
S. E. Woosley \\ Board of Studies in Astronomy and Astrophysics \\ Lick Observatory, University of California at Santa Cruz \\ Santa Cruz CA 95064 \\ and \\ Special Studies Group, Lawrence Livermore National Laboratory \\ Livermore CA 94550
}

\begin{abstract}
Presupernova models of massive stars are discussed and their explosion by either the "core bounce" or neutrino energy transport mechanism briefly reviewed. Special consideration is given to those attributes of the stellar evolution and explosion that might influence the properties of the neutron star remnant: its mass, rotation rate, magnetic field, and "kick" velocity.
\end{abstract}

\section{INTRODUCTION}

By now it is recognized that neutron stars are a likely product of the evolution of at least some massive stars. This follows from such diverse considerations as 1) the theoretical certainty that stars more massive than about $8 \mathrm{M}_{\odot}$ must evolve to a state of gravitational collapse, and barring the most massive $\left(M \gtrsim 100 \mathrm{M}_{\odot}\right.$; §2.4), produce either a black hole or a neutron star; 2) the presence of neutron stars in the remnants of historical supernovae (with records kindly provided by our hosts!) such as the Crab Nebula whose non-solar abundances, especially helium, indicate a relatively massive $\left(\sim 10 \mathrm{M}_{\odot}\right)$ progenitor; 3$)$ the distribution of pulsars with galactocentric radius (Lyne 1981) consistant with Population I objects; 4) the fact that x-ray pulsars and other forms of accreting neutron stars are frequently, though not always, found in binary systems with massive companions, (that the neutron star formed before the short lived companion died again suggests that the neutron star itself had a massive, short-lived progenitor); and 5) the masses of the binary pulsar PSR $1916+13$ which are too large to have been formed by the collapse of any but a massive star (Burrows and Woosley 1986; §4.1). Further, the inferred pulsar birth rate (Lyne 1981; this volume) is consistent with the observed rate of Type II supernovae (with conservative error bars of a factor of two on each) and the z-distribution of pulsars is consistent with birth in the Galactic disk, albeit with a large peculiar velocity.

None of these conditions requires, however, that every neutron star, or even a majority, be produced by massive stars, nor do they require, nor is it even likely, that every massive star end its life in producing a neutron star (see e.g.. Helfand and Becker 1984). The possibility of neutron star production by a massive star is, as we shall see, sensitive to the stellar mass and, unfortunately, to the physics that a given theoretician employs in his or her calculation. It turns out that the more massive the progenitor star. the less likely it is that the remnant will 
be small enough to stabilize as a neutron star. Further, it is possible to produce neutron stars by accretion at certain rates on white dwarfs in binary systems and thus bypass the need for a massive stellar parent (§3.2).

\section{TYPE II SUPERNOVAE}

Since this subject has been very recently reviewed (Woosley and Weaver 1985; 1986ab; Woosley 1986; Nomoto 1986; Wilson et al. 1986; Hillebrandt 1984, 1985), our treatment here can be terse and will concentrate only upon those aspects of the presupernova evolution and supernova mechanism that directly affect the observable properties of neutron stars, especially their mass, rotation rate, and velocity.

\subsection{Presupernova Evolution of Massive Stars}

The theoretical evolution of single massive stars allows their segregation, upon the basis of mass, into three groups, each of which encounters gravitational collapse in its own unique fashion $(\S 2.2,2.3,2.4)$. Though the existence of these three groups is clear, the precise boundaries of each are sensitive to the physics employed by various researchers, especially the initial helium abundance, the rate for the ${ }^{12} \mathrm{C}(\alpha, \gamma){ }^{16} \mathrm{O}$ reaction, the degree to which mass loss modifies the star's total mass prior to helium depletion, and the prescription for convection. Based upon a standard set of assumptions, helium abundance near $28 \%,{ }^{12} \mathrm{C}(\alpha, \gamma){ }^{16} \mathrm{O}$ as given by Caughlan et al. (1985), no mass loss, and a moderate amount of convective overshoot mixing (Weaver, Zimmerman, and Woosley 1978), Woosley and Weaver (1986ab) estimate the following mass delineations and behavior:

\subsection{Stars in the 8 to $10 M_{\odot}$ Range}

Stars above $8 \mathrm{M}_{\odot}$ will ignite carbon burning in a non-degenerate fashion, but for a relatively small interval of masses above this value the later stages of evolution continue to be complicated by effects of electron degeneracy. Stars in this mass range have been recently studied by Nomoto (1984ab; 1986; this volume), Woosley, Weaver, and Taam (1980), and Wilson et al. (1986) and their evolution is best segregated upon the basis of the helium core mass, i.e. the mass interior to the hydrogen burning shell at the time the star becomes a supernova. Main sequence stars in the 8 to $10 \mathrm{M}_{\odot}$ range end up with helium cores of roughly 2 to $3 \mathrm{M}_{\odot}$. Helium cores between about 2.2 and $2.5 \mathrm{M}_{\odot}$ develop within themselves oxygen cores that do not ignite either neon or oxygen burning in hydrostatic equilibrium. Rather the metallic cores grow to the Chandrasekhar mass (which may be decreased to $\sim 1.37 \mathrm{M}_{\odot}$ by electron capture), ignite oxygen burning at a very high central density, $\sim 2 \times 10^{10} \mathrm{~g} \mathrm{~cm}^{-3}$, and implode. The collapse occurs because degenerate oxygen burning proceeds all the way to nuclear statistical equilibrium, producing abundant iron group nuclei having low electron capture thresholds. The thermal pressure increase obtained as the temperature rises to $\sim 10^{10} \mathrm{~K}$ is inconsequential compared to the effect of the pressure lost to electron capture, and thus the core collapses even as burning continues. Explosion of these stars has been studied by 
Hillebrandt, Nomoto, and Wolff (1984) and Wilson et al. (1986) with conflicting results. The former group calculated violent hydrodynamical explosions while the latter obtained only a weaker explosion caused by neutrino energy transport ( $\$ 2.5)$.

For larger helium cores between about 2.5 and $3.0 \mathrm{M}_{\odot}$, neon, oxygen, and silicon burning do occur, albeit in complicated stages characterized by offcenter ignition, and an iron core in hydrostatic equilibrium is eventually formed (Woosley, Weaver, and Taam 1980; Nomoto 1984a, 1986). This iron core, which is near $1.40 \mathrm{M}_{\odot}$, collapses chiefly because of electron capture and, to a lesser extent, photodisintegration. Again the results of computer simulation of the core bounce and explosion are not in agreement. Hillebrandt (1982) gets a marginal explosion by hydrodynamical bounce of a $10 \mathrm{M}_{\odot}$ star, but Wilson (1985) and Burrows and Lattimer (1985) do not and Wilson et al. (1986) get an explosion based instead upon neutrino energy transport.

Stars in the 8 to $10 \mathrm{M}_{\odot}$ range all have very steep gradients in mass density just above the iron core implying a small gravitational binding energy for the external matter. Thus when an explosion does develop it always ejects all of the mass external to the iron core.

\subsection{Stars in the 10 to $\sim 70 M_{\odot}$ Range}

Stars in this mass range ignite hydrogen, helium, carbon, neon, oxygen, and silicon burning non-degenerately in hydrostatic equilibrium at their centers. The iron core thus formed collapses owing to a combination of electron capture $(M \leqslant 20$ $\left.\mathrm{M}_{\odot}\right)$ and photodisintegration $\left(\mathrm{M} \approx 20 \mathrm{M}_{\odot}\right)$. The size of the iron core at collapse becomes less sensitive to the Chandrasekhar mass as one goes to the larger mass stars characterized by higher values of central entropy. Larger central entropies have two effects that greatly influence the development of the iron core. First they allow core masses to exist in hydrostatic equilibrium that are much larger than the Chandrasekhar value. Second a larger central entropy and the shorter time scales associated with massive stellar evolution imply that the radial gradients in entropy are and remain smaller. It is these gradients which determine the extent of convective shells especially during the critical oxygen and silicon shell burning stages. The extent of the convective shells ultimately sets the mass of the iron core at collapse.

Thus there exists a main sequence mass, currently estimated at $\sim 20 \mathrm{M}_{\odot}$, above which the final iron core begins to appreciably exceed $1.4 \mathrm{M}_{\odot}$. The exact value where this occurs is of obvious great interest, all the more so because the possibility of exploding the star is very sensitive to the size of the iron core ( $\$ 2.5)$. A calculation of this critical value depends upon proper inclusion of all sources and sinks of entropy, such as neutrino losses, radiation transport, and nuclear burning, and upon the theory of convection. Somewhat surprisingly, though reasonably, the results also depend sensitively upon the carbon abundance that exists in the core following helium burning. If the carbon abundance is low, $\$ 10 \%$ by mass fraction, then carbon and neon burning never generate enough energy to surpass, even locally, that which is lost to neutrinos. Thus, insofar as entropy is concerned, the star proceeds directly and rapidly from helium exhaustion to oxygen ignition 
without ever spending time convectively burning carbon and neon in its center. Such rapid evolution allows the entropy to remain high in the inner regions and ultimately leads to large iron cores (Woosley and Weaver 1986ab).

This implies a sensitive dependence of the late stages of stellar evolution upon the carbon nucleosynthesis that occurs during helium burning. Carbon nucleosynthesis, in turn, depends in an obvious fashion upon the uncertain rate for the ${ }^{12} \mathrm{C}(\alpha, \gamma){ }^{16} \mathrm{O}$ reaction and, in a more subtle manner, upon the theories of semiconvection and convective overshoot employed in the computer code. Some degree of convective penetration beyond that radius formally unstable to convection by the Ledoux criterion is expected to occur, especially near the outer edge of the helium convective shell at a time when the central helium abundance has dropped to only a few percent and where the temperature gradient is only slightly below the critical adiabatic value. Mixing by convective overshoot during this stage brings new helium into a region in which the oxygen to carbon ratio is already high. Since the formation rate of carbon depends upon the cube of the $\alpha$-particle abundance while rate of its conversion to ${ }^{16} \mathrm{O}$ is linear, each new helium nucleus brought in almost invariably converts a carbon nucleus to an oxygen nucleus. Thus the carbon nucleosynthesis is sensitive to how much overshoot mixing is assumed.

The models calculated by Woosley and Weaver (1986b), Wilson et al. (1986), and Weaver, Woosley, and Fuller (1985) all assume the overshoot parametrization discussed by Weaver, Zimmerman, and Woosley (1978). That is, single zones bounding regions that are formally unstable to convection by the Ledoux criterion are treated as semi-convective. Though no convective energy transport occurs, the composition of this boundry zone is slowly mixed into the convective helium core. The diffusion coefficient for this "overshoot zone" is calculated in the same fashion as in an ordinary convective zone, but the temperature gradient used in the convective velocity calculation is some adjustable fraction larger than the adiabatic one. In all calculations thus far the value of this constant is $1 \%$, though sensitivity studies using other smaller values are currently underway. It is believed that any non-trivial value for this parameter will give similar results. The diffusion coefficient for these special "overshoot" zones is further restricted, as it is in all semi-convective zones, to be no greater than $10 \%$ of the radiative diffusion coefficient (see Weaver, Zimmerman, and Woosley 1978 for further discussion and justification).

If there were no overshoot mixing, the carbon abundance would be higher and it would be easier to form iron cores of near the Chandrasekhar mass for larger values of main sequence mass. If a greater amount of overshoot mixing were employed, the iron cores would be larger for smaller stars. Calculations to quantify these statements are in progress.

\subsection{Stars Heavier Than $70 M_{\odot}$}

Stars that have helium core masses larger than $32 \mathrm{M}_{\odot}$ at the end of helium burning become unstable as they evolve to more advanced burning stages. For the present ${ }^{12} \mathrm{C}(\alpha, \gamma){ }^{16} \mathrm{O}$ rate and parametrization of convection, the carbon and neon abundances are too small to provide exoergic burning stages powered by these fuels. 
Thus the central nuclear energy generation does not exceed neutrino losses until oxygen begins to burn. Oxygen ignition occurs at relatively high values of central entropy $(S / k \geq 7)$ and temperature which favor the production of electron-positron pairs. Production of the pair rest mass temporarily reduces the structural $\Gamma$ of the star below 4/3 and collapse begins (Barkat, Rakavy, and Sack 1967). What follows depends very sensitively upon the initial entropy, and hence mass, of the helium core.

For helium core masses greater than $\sim 50 \mathrm{M}_{\odot}$, with the precise value still awaiting determination, the instability is violent enough that the core collapses to between 3.5 and $5 \times 10^{9} \mathrm{~K}$, burns a fraction of the oxygen core to silicon and heavier elements, and, for helium cores lighter than about $120 \mathrm{M}_{\odot}$, produces enough energy to reverse the implosion and completely disrupt the star (Woosley and Weaver 1982; Bond, Arnett, and Carr 1984; Ober, El Eid, and Fricke 1983; Glatzel, El Eid, and Fricke 1985; El Eid and Langer 1986). The light curves from such explosions can be very brilliant, especially towards the heavier end of this mass range, since a great deal of radioactive ${ }^{56} \mathrm{Ni}$ is produced in the explosion. For helium cores still more massive than $\sim 120 \mathrm{M}_{\odot}$, the kinetic energy of infall becomes too great to be reversed by a nuclear explosion (unless one includes the effect of rotation which can raise the limit considerably; Stringfellow, Woosley, and Bodenheimer 1983; Glatzel, El Eid, and Fricke 1985). As the nuclear burning passes from oxygen burning to silicon burning and finally nuclear statistical equilibrium, reactions begin to absorb more energy than they release. The star collapses directly to a black hole (Woosley, Wilson, and Mayle 1986). Thus one way or another, helium cores heavier than about $50 \mathrm{M}_{\odot}$ (i.e., stars heavier than about $110 \mathrm{M}_{\odot}$ on the main sequence) avoid neutron star production.

For helium cores between 32 and $\sim 50 \mathrm{M}_{\odot}$, the pair instability manifests itself as an extreme form of pulsational instability and does not lead directly to total stellar disruption. Main sequence stars of $75 \mathrm{M}_{\odot}$ and $100 \mathrm{M}_{\odot}$ studied by Woosley and Weaver $(1986 \mathrm{~b})$ produced helium cores of 36 and $45 \mathrm{M}_{\odot}$ respectively (Table I) which became violently unstable at oxygen ignition: The $45 \mathrm{M}_{\odot}$ core, whose instability was followed in some detail, ejected about $3 \mathrm{M}_{\odot}$ with total kinetic energy $4 \times 10^{50}$ erg over the course of 4 "pulsations." Substantial cooling by neutrino losses during the Kelvin-Helmholtz stage following each outward excursion reduced the entropy sufficiently that stable silicon burning was finally ignited in the core. Eventually an iron core of $2.3 \mathrm{M}_{\odot}$ was produced which collapsed on the photodisintegration instability (Table I). Whether neutrino energy transport can eject the matter external to a core of this size is still to be determined although Wilson et al. (1986) studied a similar core and found growth to over $3 \mathrm{M}_{\odot}$ without explosion. The possibility of an explosion energized by rotation and nuclear burning (Bodenheimer and Woosley 1983) also needs to be examined for this configuration. But one way or another, it seems likely that stars in the pulsationally unstable regime will also leave black hole remnants, not neutron stars. 
Table I. Presupernova Models and Explosions

\begin{tabular}{|c|c|c|c|c|c|c|}
\hline $\begin{array}{l}\text { Main } \\
\text { Seq. } \\
\text { Mass }\end{array}$ & $\begin{array}{c}\text { Helium } \\
\text { Core } \\
\text { Mass }\end{array}$ & $\begin{array}{l}\text { Iron } \\
\text { Core } \\
\text { Mass }\end{array}$ & $\begin{array}{c}\text { Expl. } \\
\text { Energy }^{a} \\
\left(10^{50} \mathrm{erg}\right)\end{array}$ & $\begin{array}{c}\text { Residual } \\
\text { Baryon } \\
\text { Mass }^{a}\end{array}$ & $\begin{array}{c}\text { Neutron } \\
\text { Star } \\
\text { Mass }^{a}\end{array}$ & $\begin{array}{l}\text { Heavies } \\
\text { Ejected } \\
(\mathrm{Z} \geq 6)\end{array}$ \\
\hline 11 & 2.4 & $-b$ & 3.0 & 1.42 & 1.31 & $\sim 0$ \\
\hline 12 & 3.1 & 1.31 & 3.8 & 1.35 & 1.26 & 0.96 \\
\hline 15 & 4.2 & 1.33 & 2.0 & 1.42 & 1.31 & 1.24 \\
\hline 20 & 6.2 & 1.70 & - & - & - & 2.53 \\
\hline 25 & 8.5 & 2.05 & 4.0 & 2.44 & 1.96 & 4.31 \\
\hline 35 & 14 & 1.80 & - & - & - & 9.88 \\
\hline 50 & 23 & 2.45 & - & - & - & 17.7 \\
\hline 75 & 36 & ${ }^{c}$ & - & - & BH? & $30 ?$ \\
\hline 100 & 45 & $\sim 2.3^{c}$ & $z 4$ & - & BH? & $39 ?$ \\
\hline
\end{tabular}

${ }^{a}$ All except for $100 \mathrm{M}_{\odot}$ determined by Wilson et al. (1986)

${ }^{b}$ Never developed iron core in hydrostatic equilibrium

${ }^{c}$ Pulsational pair instability at oxygen ignition

\subsection{Core Collapse and Explosion in $M \leqslant 70 M_{\odot}$}

For many years the outward propagation of the shock wave generated by the bounce of the collapsing iron core of stars in this mass range has been studied in the hope that it might provide the energy required to eject the stellar mantle and envelope and produce a supernova. Unfortunately the most careful theoretical studies are still at variance regarding the success of this and other possible mechanisms, e.g. neutrino energy transport.

To summarize briefly, the shock wave generated by core bounce in a massive star is born with a certain characteristic energy $\left(\sim 7 \times 10^{51} \mathrm{erg}\right.$; Burrows and Lattimer 1983) deep within the interior, but not at the center, of the neutronized core. Typically the mass interior to the point where the shock first develops is $\sim 0.8$ $\mathrm{M}_{\odot}$. As it moves outwards this shock experiences energy losses, principally owing to photodisintegration of heavy nuclei and, once the density falls below a few times $10^{11} \mathrm{~g} \mathrm{~cm}^{-3}$, to neutrino losses. The larger the mass of the collapsing iron core, the greater are the losses, especially photodisintegration which takes about $1.5 \times 10^{51}$ erg for each $0.1 \mathrm{M}_{\odot}$ of core material that must.be traversed. For iron core masses greater than 1.25 to $1.40 \mathrm{M}_{\odot}$, with the precise value depending upon choice of nuclear equation of state and the distribution of entropy and electron fraction, the shock wave dies before reaching the edge of the iron core. A supernova powered by hydrodynamical energy transport then becomes impossible for larger cores.

This range marginally intersects smaller core masses given in Table I and, not surprisingly, various researchers have calculated qualitatively different results for the core bounce. Baron, Cooperstein, and Kahana (1985ab) obtain energetic 
explosions of 12 and $15 \mathrm{M}_{\odot}$ model stars but employ an equation of state that is softer, hence more favorable to explosion, than traditional in the nuclear physics community (though Baron et al. 1985 defend their choice as natural for the special environment appropriate to supernova cores). Other researchers have not yet attempted confirmation of the Baron calculations though work is in progress by Wilson and Mayle. Presently it appears marginally possible that stars in the 8 to $\sim 20 \mathrm{M}_{\odot}$ range will explode promptly. If so, the time scale for explosion following maximum compression is $\sim 20 \mathrm{~ms}$ and the explosion energy, around $10^{51} \mathrm{erg}$.

If these stars do not explode promptly, and certainly the more massive cores in Table I will not, then the outgoing shock stalls and becomes an accretion shock. Hope for exploding the star now hinges upon the efficiency of neutrinos in transporting a sufficient fraction of the core binding energy to a region that is simultaneously sufficiently optically thick to neutrinos and insufficiently bound by gravity that thermal overpressure can lead to its ejection. Wilson and coworkers have presented calculations (Wilson 1985; Wilson et al. 1986; Mayle 1986) and arguments (Bethe and Wilson 1985) to show that such a mechanism can indeed produce eventual explosion in all save the most massive stellar cores. The mechanism depends upon the capture over a long period of time, typically several hundred milliseconds, of neutrinos by matter directly beneath the accretion shock. Only a few percent of the neutrinos flowing through are captured. This revives the outward motion of the shock, eventually giving rise to ejection of all external matter and explosion energies in the range $\sim 3 \times 10^{50}$ to $10^{51} \mathrm{erg}$. Two points are of special interest here. First the possibility of a succesful explosion that leaves behind a baryonic mass of over $2.4 \mathrm{M}_{\odot}$ (Table I) implies that some supernovae could leave black hole (or at least very massive neutron star) remnants. The remnants would spend several seconds, perhaps even a minute before losing enough neutrinos to collapse inside their event horizon. Second, during the relatively long period that the "delayed explosion" mechanism requires for its operation, a substantial quantity of matter may accrete onto the neutronized core. Thus the core that is left behind may be larger than the original iron core that collapsed. For the lower energy explosions some of the inner mantle material may end up moving slower than the escape velocity and fall back onto the neutron star, again increasing the probability of massive neutron star or black hole formation.

The neutrino energy transport model of Wilson has yet to be verified by other researchers and, as Arnett discusses in this volume, remains quite controversial. In order to produce a more energetic and less marginal explosion, one needs to boost the neutrino flux coming out of the core. This might be achieved by either a global overturn of the core (Epstein 1979; Colgate and Petschek 1980) or by a more docile form of convection (Mayle 1986; Arnett 1986, this volume) which would bring neutrinos trapped deeper in the core to the neutrinosphere, thereby increasing the neutrino luminosity of the star at a critical time. 


\section{OTHER SYSTEMS THAT MIGHT LEAVE NEUTRON STARS}

\subsection{Type Ib and "Type III" Supernovae}

The name "Type II Supernova" refers specifically to a supernova whose spectrum displays lines of hydrogen. It is quite probable that very massive single stars $(M$ $z 40 \mathrm{M}_{\odot}$ ), or some less massive stars in interacting binary systems, end their lives devoid of a hydrogen envelope. Such stars would exhibit bolometric and spectroscopic properties quite distinct from Type II supernovae despite identical explosion mechanisms operating in the core. I have recently calculated (Woosley 1986; Ensman and Woosley 1986) the light curves of the helium cores of the 15 and $25 \mathrm{M}_{\odot}$ models of Table I, but with the hydrogen envelopes artificially removed. The light curves are totally dominated by energy from radioactive decay $\left({ }^{56} \mathrm{Ni}\right.$ and ${ }^{56} \mathrm{Co}$ ) and are qualitatively similar to those calculated for Type I supernovae (Woosley and Weaver 1986ab). They are, however, broader (FWHM $\sim 70 \mathrm{~d}$ for the $25 \mathrm{M}_{\odot}$ star; $8 \mathrm{M}_{\odot} \mathrm{He}$ core $)$ and dimmer at peak light $\left(L_{\max }=3.4 \times 10^{42} \mathrm{erg} \mathrm{s}^{-1}\right)$ than a typical Type Ia supernova. These are also properties attributed to a recently discovered subclass of "Type I" supernovae called Type Ib (Wheeler and Leverault 1985; Gaskell et al. 1986) or sometimes Type III (Chevalier 1986). Other proposed examples of this class are SN $1985 \mathrm{f}$ (the "Filippenko-Sargent object," Filippenko and Sargent 1985, 1986) and Cas A. While I do not at the present wish to join the bulk of the community in attributing Type $\mathrm{Ib}$ supernovae to massive stellar cores (because of reservations I have regarding the spectrum at peak light; see also Branch and Nomoto 1986), it is worth noting the probable existence of a class of supernovae that 1) lack hydrogen, 2) are associated with a massive stellar population, 3) may have been surrounded by envelopes from pre-explosive mass loss, 4) have a light curve powered totally by radioactivity, 5) are optically dim compared to Type Ia, and 6) unlike Type Ia, may leave neutron star or black hole remnants.

\subsection{Accreting White Dwarfs}

It has long been speculated that continued accretion onto a white dwarf star might push that star over the Chandrasekhar mass and cause collapse directly to a neutron star. If this occurred a neutron star would be formed in a binary system (although see $\S 4.3$ ) in an event that might be very difficult to detect. Lacking both hydrogen envelope and substantial ejection of ${ }^{56} \mathrm{Ni}$ during the explosion, the collapse would be optically dim, hence the term "silent supernova" (Nomoto 1984c).

Historically the problem in producing such events has been avoiding the total disruption of the white dwarf by a degenerate carbon explosion. Recently, however, several scenarios have emerged that circumvent this difficulty. All utilize white dwarfs which, for one reason or another, have zero or trivially small carbon abundances in their centers as they approach the Chandrasekhar mass.

Canal and Isern (Canal and Schatzman 1976; Canal and Isern 1979; Isern et al. 1983) have proposed a phase transition leading to a mechanical separation of carbon and oxygen in systems with very low accretion rates. By the time the 
Chandrasekhar mass is reached, the center of the star consists almost entirely of oxygen. Ignition of nuclear reactions is thus forestalled until such high densities are reached $z 10^{10} \mathrm{~g} \mathrm{~cm}^{-3}$ that electron capture leads to collapse (see $\S 2.1$ ) much as in a $2.2 \mathrm{M}_{\odot}$ helium star.

Saio and Nomoto (1985), Nomoto (1986), Woosley and Weaver (1985), and Nomoto and Iben (1985) have discussed an alternate scheme wherein the merging of two carbon-oxygen white dwarfs accomplished by gravitational radiation leads to such high accretion rates that carbon burning is ignited non-degenerately offcenter. A diffusive burning front propagates to the center of the star while it remains in hydrostatic equilibrium, depleting the star of carbon and converting the inner regions to oxygen and neon. Continued accretion then leads to oxygen ignition and collapse of the white dwarf as above.

Finally one may just start with a neon-oxygen white dwarf, the product of an 8 to $10 \mathrm{M}_{\odot}$ star that has lost its envelope in a binary system, and accrete on that object until it collapses. Observational evidence for white dwarfs of this type in accreting binary systems has been recently reviewed by Starrfield, Sparks, and Truran (1986) within the context of classical novae.

\section{NEUTRON STAR PROPERTIES}

\subsection{Masses}

From the considerations of the previous sections and references therein it follows that neutron stars should be born with a variety of masses (see e.g. Rappaport and Joss 1981). In particular, there is no reason why the preferred mass should be near $1.44 \mathrm{M}_{\odot}$. In the simplest case imaginable where a white dwarf is pushed over its critical mass (Saio and Nomoto 1985), the collapse starts with a mass closer to $1.42 \mathrm{M}_{\odot}$ because of previous electron capture and because a central density of only $10^{10} \mathrm{~g} \mathrm{~cm}^{-3}$ need be achieved in order to trigger collapse. More importantly, the binding energy of the final neutron star must be subtracted off from this mass (e.g. Burrows and Woosley 1986), the difference being the neutrinos emitted during the collapse. The specific amount subtracted depends upon the nuclear equation of state adopted but is near $10 \%$. Thus the gravitational mass of the neutron star would be near $1.3 \mathrm{M}_{\odot}$, possibly $1.35 \mathrm{M}_{\odot}$.

Somewhat smaller masses might be achieved from the collapse of massive stellar cores, especially in the 8 to $20 \mathrm{M}_{\odot}$ range. There, depending upon the preexplosive electron capture and explosion mechanism, the residual iron core mass might be as small as $\sim 1.35 \mathrm{M}_{\odot}$, (e.g. Baron, Cooperstein, and Kahana 1985ab; Wilson et al. 1986), thus yielding a neutron star mass near $1.25 \mathrm{M}_{\odot}$. General considerations of shock wave strength (Burrows and Lattimer 1983; Hillebrandt, Nomoto, and Wolff 1984; Baron, Cooperstein, and Kahana 1985ab) suggest that if the iron core became smaller than $\sim 1.25 \mathrm{M}_{\odot}$ the shock wave would begin to blow away a significant part of the neutron star. This is strictly forbidden in all but very rare events by nucleosynthetic considerations (Hartmann, Woosley, and El Eid 1985). It is almost certainly impossible to get a stellar core smaller than this size to collapse anyway. Photodisintegration would be negligible and the central 
density would be too low to cause collape by electron capture. Subtracting at most $10 \%$ for the binding energy gives a stringent lower limit to the neutron star mass of about $1.15 \mathrm{M}_{\odot}$. As discussed above a more likely value would be $1.3 \mathrm{M}_{\odot}$.

The largest neutron star mass is given entirely by considerations of nuclear equation of state since the evolution of massive stars is capable of providing a continuum of masses up to and above the maximum value. The more massive neutron stars should be rarer owing to the decreasing abundance of stars of higher mass. Adopting as a representative example a critical mass near $20 \mathrm{M}_{\odot}$ as the heaviest star for which the entropy is low enough to allow convergence of the iron core mass to near the Chandrasekhar value, a value of $40 \mathrm{M}_{\odot}$ as the heaviest star that leaves a neutron star remnant rather than a black hole, and $8 M_{\odot}$ for the lowest mass star that undergoes gravitational collapse, one finds (Miller and Scalo 1979) that about $10 \%$ of neutron stars should have masses appreciably in excess of the typical 1.3 $\mathrm{M}_{\odot}$ value. This number is insensitive to the assumption of $40 \mathrm{M}_{\odot}$ for those stars that produce black holes (unless the value becomes much smaller), but is very sensitive to the assumed 8 and $20 \mathrm{M}_{\odot}$ values as well as the IMF of Miller and Scalo. This number should therefore be treated as a rough approximation. Of course one must also consider the possibility that not all neutron stars have a massive parent (§3.2).

A case of special interest is, PSR $1913+16$, the first discovered of the binary pulsars for which the masses of both components have now been determined to high accuracy (Weisberg and Taylor 1984; Taylor 1985, Taylor, this volume). Recent analyses give $1.444 \mathrm{M}_{\odot}$ and $1.384 \mathrm{M}_{\odot}$ for the two stars, both of which are apparently neutron stars (Srinivasan and Van den Heuvel 1982) and the heavier of which is the pulsar. From the above discussion, neither the pulsar nor its companion could have evolved from a collapsed white dwarf unless accretion continued long after the collpase of the neutron star that became the pulsar. The latter is probably ruled out by the young age of the pulsar and, in any event, one is still left with the large mass of the companion. Burrows and Woosley (1986) present arguments to show that this binary pulsar probably evolved from a system of two massive stars, both having main sequence masses between about 15 and $20 \mathrm{M}_{\odot}$. If so, a "kick velocity" of $\sim 200 \mathrm{~km} \mathrm{~s}^{-1}$ is required if the system is to remain gravitationally bound following the second explosion.

\subsection{Rotation Rate and Magnetic Field}

Little in the way of quantitative predictions can presently be made, or are likely to be made by theoreticians in the near future, regarding these important attributes of young neutron stars. Upper main sequence stars earlier than F5 are well known to have high equatorial rotational velocities. Assuming that these stars rotate uniformly, the inferred total angular momentum, $J$, is very large, the dimensionless quantity $c J / G M^{2}$ lying in the range 9 to 18 (Kiguchi and Sato 1985). If angular momentum were conserved and not transported, rotation would unavoidably play a major role during the final stages of stellar evolution. The core would evolve to the point where the ratio of rotational energy to gravitational energy $(T / W)$ exceeded about $25 \%$ and triaxial deformation would then ensue on a dynamical 
time scale. Bifurcation and/or rapid transport of angular momentum, perhaps by gravitational radiation, would occur and ultimately a neutron star would be born which, neglecting the effects of magnetic interaction (?), would rotate at a substantial fraction of breakup speed. From the standpoint of stellar evolution there is no apparent fundamental reason why this should not occur.

However, angular momentum transport will occur throughout the stellar lifetime, if by no means other than convection. Endal and Sofia $(1976 ; 1978)$ using one parametrization of angular momentum transport found that rotation would not become a major effect during hydrogen, helium, or carbon burning in a $10 \mathrm{M}_{\odot}$ star, but extrapolated that rotation would become important during later stages. These later stages will be convective however, at least in stars more massive than $\sim 10 \mathrm{M}_{\odot}$. The several stages of convective oxygen and silicon shell burning could transport substantial angular momentum out of the inner several solar masses, causing the core to tend towards solid body rotation. A large velocity shear near the edge of this core could lead to turbulent dissipation or magnetic braking.

Beyond these already speculative comments it is difficult to say more. Core contraction from the onset of dynamical instability $\left(\rho_{\text {cent }} \sim 10^{9}\right.$ to $10^{10} \mathrm{~g}$ $\mathrm{cm}^{-3}$ ) should proceed rapidly enough that, unless triaxial deformation develops, angular momentum is conserved. In particular the viscosity afforded by escaping neutrinos should be negligible (Lindblom and Detweiler 1979). No reason is readily apparent why the rotation rate of the young neutron star should be any. particular number (other than the limiting value required for triaxial deformation) or why the magnetic field should tend to lie in the range $10^{12}$ to $10^{13}$ gauss, though such fields are easily attainable if flux is conserved and the silicon core has a magnetic field similar to that of a magnetic white dwarf (e.g. Am Herc).

It is worth noting, however, that different scenarios for producing neutron stars might reasonably lead to different ranges of rotation rates and magnetic fields. A collapsing white dwarf in a merging binary pair might be very rapidly spun up to near breakup (Nomoto 1986). A somewhat more distant binary pair might lead to the collapse of a white dwarf (a presumed rigid rotator) that is tidally locked to the period of the binary, thus a more slowly rotating neutron star. Stars in the 8 to $10 M_{\odot}$ range will not experience oxygen and silicon burning in hydrostatic equilibrium. The last convective phase of many of these stars is carbon burning at a central density of $10^{5} \mathrm{~g} \mathrm{~cm}^{-3}$. Yet in more massive stars convection during silicon burning occurs up to central densities of several times $10^{8} \mathrm{~g} \mathrm{~cm}^{-3}$, thus affording the possibility of angular momentum transport out of a core that is already quite compact.

Obviously almost any observed rotation rate or classes of rotation rates can be accommodated.

\section{3 "Kick" Velocities}

Pulsars are born having a relatively high peculiar velocity, typically on the order of a few hundred kilometers per second. This could be due to escape from a binary pair or could reflect some asymmetry in the explosion itself. Kick velocities of the same 
order may be required in order to keep those few binary pulsars that are observed gravitationally bound to their companions. Since no theoretical simulations of core collapse and supernova explosion have been carried out in three dimensions any comments one would make on this subject must presently be of a speculative nature. Still we note several attributes of the explosion mechanism that might easily lead to a recoil. The required effect is only at the level of several percent in the supernova energy.

Most calculations of core bounce carried out so far have used 1D hydrodynamics codes and, of necessity, generate radially symmetric results, but even these studies are useful for showing that a large fraction of the core, that fraction external to the "homologous core" $\left(\sim 0.6\right.$ to $\left.0.8 \mathrm{M}_{\odot}\right)$ is collapsing supersonically when the center of the star reaches nuclear density (e.g. Baron, Cooperstein, and Kahana 1985ab). Thus, during core collapse and bounce, large regions of the star are, for the first time since its formation, out of sonic communication with one another. Any asynchronism between collapse onset or difference in collapse rate between two sides of the core will result in one side bouncing slightly in advance of the other. Then the shock wave that moves out will not have radial symmetry but will be egg-shaped, with its leading edge along the angle that bounced first: This egg-shaped shock could be decomposed into a central radially symmetric shock and a smaller one, originating off-center. It is the small off-center component that gives an asymmetric momentum or "kick" to the explosion.

Alternatively one may consider a "delayed" explosion driven by neutrino energy transport (Wilson et al. 1986). In this model the explosion occurs at late times owing to the capture by material behind a stalled accretion shock of a fraction of the neutrino luminosity coming from the mantle and outer layers of the young neutron star. There is no reason that this flux must be precisely the same on both sides of the core. Once again disjoint regions are out of communication. An asymmetry in the neutrino luminosity of a few percent can be envisioned, if not easily calculated.

Thus it seems reasonable, though perhaps not necessary, that the neutronized core acquire some peculiar velocity as a result of small but fundamental asymmetries in the energizing mechanism of the explosion. In the case of the core bounce mechanism, the non-radially symmetric hydrodynamics should ultimately translate into an angular asymmetry of the momentum of the ejecta of comparable magnitude (properly scaled for mass). In the neutrino transport model or during neutrino cooling following a sucessful core bounce, the asymmetry could be contained in the momentum distribution of the neutrinos themselves. The effective energy in the neutrinos is, after all, $\sim 10 \%$ of the rest mass of the neutron star. Thus a non-radial asymmetry.in neutrino momentum distribution of only $1 \%$ would give the neutron star a recoil velocity of a few hundred kilometers per second. Such an asymmetry in total neutrino momentum could be a consequence of inhomogeneous composition and neutrino opacity or, especially, of large scale convective motions in the core during the first few seconds of its existence (Epstein 1979; Bruenn, Buchler, and Livio 1979; Colgate and Petschek 1980; Livio, Buchler and Colgate 1980; Smarr et al. 1981; Wilson et al. 1986). 
The overall lesson that stellar evolution brings to those studying neutron stars is one of great possible diversity in the initial properties.

This work has been supported at UCSC by the National Science Foundation (AST-84-18185) and, at LLNL, by the Department of Energy through contract number W-7405-ENG-48. The author is also grateful for a faculty research grant form UCSC to cover a portion of the expenses incurred in attending the meeting.

\section{REFERENCES}

Arnett, W. D. 1986, Preprint Enrico Fermi Inst., Univ. Chicago, submitted to Ap. J. Lettr.

Barkat, Z., Rakavy, G., and Sack, N. 1967, Phys. Rev. Lettr. 19, 379.

Baron, E., Cooperstein, J., and Kahana, S. 1985a, Phys. Rev. Lettr., 55, 126. . 1985b, Nucl. Phys., A440, 744.

Baron, E., Brown, G. E., Cooperstein, J., and Prakash, M. 1985, Preprint.

Bethe, H. A., and Wilson, J. R. 1985, Ap. J., 295, 14.

Bisnovatyi-Kogan, G. S. 1971, Sov. Astron. A. J., 14, 652.

. 1980, Ann. N. Y. Acad. Sci., 336, 389.

Bisnovatyi-Kogan, G. S. , Popov, Y. P., and Samochin, A. A. 1976, Ap. and Spac. Sci,. 41, 287.

Bodenheimer, P., and Woosley, S. E. 1983, Ap. J., 269, 281.

Bond, J. R., Arnett, W. D., and Carr, B. J. 1984, Ap. J., 280, 825.

Branch, D. and Nomoto, K. 1986, preprint submitted to Astron. and Ap. Letters.

Bruenn, S. W., Buchler, R. J., and Livio, M. 1979, Ap. J. Lettr., 234, L183.

Burrows, A. and Lattimer, J. M. 1983, Ap. J., 270, 735. . 1985, Ap. J., 299, L19.

Burrows, A. and Woosley, S. 1986, Ap. J., in press.

Canal, R., and Schatzman, E. 1976, Astron. and Ap., 46, 229.

Canal, R., and Isern, J. 1979, in White Dwarfs and Variable Degenerate Stars, Proc. IAU Colloq. 53, ed. H. M. Van Horn and V. Weidemann, Univ. of Rochester, p. 52.

Caughlan, G. R., Fowler, W. A., Harris, M. J., and Zimmerman, B. A. 1985, Atomic Data and Nuclear Data Tables, 32, 197.

Chevalier, R. A. 1986, in Highlights of Astronomy, in press. 
Colgate, S. A., and Petschek, A. G. 1980, Ap. J. Lettr., 236, L115.

El Eid, M. F., and Langer, N. 1986, Astron. and Ap., in press.

Endal, A. S., and Sofia, S. 1976, Ap. J., 210, 184. . 1978, Ap. J., 220, 279.

Ensman, L. and Woosley, S. E. 1986, in preparation for Ap. J.

Epstein, R. 1979, M. N. R. A. S., 188, 305.

Filippenko, A. V., and Sargent W. L. W. 1985, Nature, 316, 407. . 1986, Astron. J., in press.

Gaskell, C. M., Cappellaro, E., Dinerstein, H. L., Garnett, D., Harkness, R. P., and Wheeler, J. C. 1986, preprint Univ. Texas, Austin, submitted to Ap. J.

Glatzel, W., El Eid, M. F., and Fricke, K. J. 1985, Astron. and Ap., 149, 413.

Hartmann, D. H., Woosley, S. E., and El Eid, M. 1985, Ap. J., 297, 837.

Helfand, D. J., and Becker, R. H. 1984, Nature, 307, 215.

Isern, J., Labay, J., Hernanz, M. and Canal, J. R. 1983, Ap. J., 273, 320.

Hillebrandt, W. 1982, Astron. and Ap. Lettr., 110 L3. . 1984, Ann. N. Y. Acad. Sci, 422, 197.

. 1985, To appear in Cosmogonical Processes, proceedings of A. G. W. Cameron's 60th Birthday symposium, Boulder, Colorado.

Hillebrandt, W., Nomoto, K., and Wolff, R. G. 1984, Astron. Ap. 133, 175.

Lindblom, L., and Detweiler, S. 1979, Ap. J. Lettr., 232, L101.

Livio, M. J. R., Buchler, R. A. and Colgate, S. A. 1980, Ap. J. Lett., 238, L139.

Lyne, A. G. 1981, in Supernovae: A Survey of Current Research, ed. M. J. Rees and R. J. Stoneham, (D. Reidel: Dordrecht), p. 405.

Kiguchi, M., and Sato, K. 1985, preprint, Univ. Tokyo, UTAP-30.

LeBlanc, J. M., and Wilson, J. R. 1970, Ap. J., 161, 541.

Mayle, R. 1986, Ph.D. thesis, UC Berkeley.

Meier, D. L., Epstein, R. I., Arnett, W. D., and Schramm, D. N. 1976, Ap. J., 204, 869 .

Miller, G. E., and Scalo, J. M. 1979, Ap. J. Suppl., 41, 513.

Müller, E., and Hillebrandt, W. 1979, Astron. and Ap., 80, 147.

Nomoto, K. 1984a, Ap. J., 277, 791.

. 1984b, The Crab Nebula and Related Supernova Remnants, ed. M. Kafatos and R. B. C. Henry, Cambridge Univ. Press: Cambridge. In press. 
. 1984c, Problems of Collapse and General Relativity, ed. D. Bancel and M. Signore, (D. Reidel: Dordrecht), p. 89.

- 1986, Proceedings of the 12th Texas Symposium on Relativistic Astrophysics, Ann. N. Y. Acad. Sci., in press.

Nomoto, K., and Iben, I. Jr. 1985, Ap. J., 297, 531.

Ober, W., El Eid, M. F., and Fricke, K. J. 1983, Astron. and Ap., 119, 61.

Ohnishi, T. 1983, Technical Reports of the Institute of Atomic Energy, Kyoto Univ., No. 198.

Rappaport, S., and Joss, P. C. 1981, in X-Ray Astronomy With the Einstein Satellite,ed. R. Giaconni (D. Reidel: Dordrecht), p. 123.

Saio, H., and Nomoto, K. 1985, Astron. and Ap., 150, L21.

Smarr, L., Wilson, J. R., Barton, R. T., and Bowers, R. L. 1981, Ap. J., 246, 515.

Srinivasan, G., and Van den Heuvel, E. P. J. 1985, Astron. and Ap., 108, 143.

Stringfellow, G. S., Woosley, S. E., and Bodenheimer, P. 1983, Bul. Am. Astron. Soc., 15, 955 and in preparation.

Starrfield, S., Sparks, W. M., and Truran, J. W. 1986, Univ. of Illinois preprint IAP-86-30, Ap. J. Lettr., in press.

Taylor, J. H. 1985, private communication.

Weaver, T. A., Zimmerman, G . B., and Woosley, S. E., 1978, Ap. J., 225, 1021.

Weaver, T. A., Woosley, S. E., and Fuller, G. M. 1985, Numerical Astrophysics, ed. J. Centrella, J. LeBlanc, and R. Bowers. Jones and Bartlett: Boston. p. 374

Weisberg, J. M., and Taylor, J. H. 1984, Phys. Rev. Lettr., 52, 1348.

Wheeler, J. C., and Leverault, R. 1985, Ap. J. Lettr., 294, L17.

Wilson, J. R. 1985, Numerical Astrophysics, ed. J. M. Centrella, J. M. LeBlanc, and R. L. Bowers, (Jones and Bartlett: Boston), p. 422.

Wilson, J. R., Mayle, R., Woosley, S. E., and Weaver, T. A. 1986, Proc. of Twelfth Texas Symp. on Rel. Ap., Ann. N. Y. Acad. Sci., in press

Woosley, S. E. 1986, in Nucleosynthesis and Chemical Evolution, Proc. 16th Advanced Course, Swiss Society of Astronomy and Astrophysics, Saas-Fee 1986, ed. B. Hauck and A. Maeder, (Geneva Observatory: Switzerland), in press.

Woosley, S. E., and Weaver, T. A. 1982, Supernovae: A Survey of Current Research, ed. M. J. Rees and R. J. Stoneham, D. Reidel: Dordrecht, p. 79

- 1985, Nucleosynthesis and Its Implications On Nuclear and Particle Physics, Proc. Fifth Moriand Astrophysics Conference, ed. J. Audouze and N. Mathieu, (D. Reidel: Dordrecht), p. 145.

. 1986a, Ann. Rev. Astron. and Ap., in press. 
- 1986b, in Radiation Hydrodynamics in Stars and Compact Objects, Proc. IAU Colloq. 89, Copenhagen 1985, ed. D. Mihalas and K. H. Winkler.

Woosley, S. E., Weaver, T. A., and Taam, R. E. 1980, Type I Supernovae, ed. J. C. Wheeler, Austin: Univ. of Texas, p. 96

Woosley, S. E., Wilson, J. R., and Mayle, R. 1986, Ap. J., 302, 19.

\section{DISCUSSION:}

F. Seward: What is the minimum mass for a neutron star made during the collapse you have described?

Woosley: As I have discussed in $\S 4.1$, the minimum mass for the core is $\sim 1.25$ $\mathrm{M}_{\odot}$. After subtracting off the binding energy, the smallest neutron star mass is $1.15 \mathrm{M}_{\odot}$. More typical values should be near $1.3 \mathrm{M}_{\odot}$.

Z. W. Li: What is the effect of the [nuclear] equation of state on the calculation of SN II? Is it important?

Woosley: For those stars having sufficiently small iron cores that a hydrodynamical explosion might be possible, the nuclear equation of state is important in giving the explosion energy for a given core mass and for determining the maximum core mass that can explode by the mechanism. As Baron, Cooperstein, and Kahana $(1985 \mathrm{ab})$ have shown, an equation of state that is "softer" at high density is more favorable to explosion.

A. Burrows: [A comment and a question] I believe that the fatter iron cores that are destined to form black holes will do so in seconds, not minutes. The mass accretion rate through the shock should be large enough to quickly put the core over the critical mass. Can you estimate the amount of ${ }^{56} \mathrm{Ni}$ produced in Type II's as a function of progenitor mass?

Woosley: Bodenheimer and I (1983) studied the continued collapse of the core in a $25 \mathrm{M}_{\odot}$ model which had failed to explode. We found that the collapsed remnant grew to $3.8 \mathrm{M}_{\odot}$ in about 20 seconds. Thus I agree that, excluding large amounts of rotation, the mass will increase above any value allowing a final stable neutron star within much less than one minute as you say. The real issue however, is the time scale for removing so much entropy from the remnant that it cannot be supported by thermal pressure. Those calculations remain to be done in an object that is becoming a black hole and including convection, though I know you are working on just such a study. All in all, a time scale of 10 seconds (to a factor of several) seems reasonable.

The ${ }^{56} \mathrm{Ni}$ synthesis depends upon the explosion energy and, more importantly on the pre-explosive density gradient surrounding the core. The shallower the gradient the more material stands near the "bomb" and gets heated above the $5 \times 10^{9} \mathrm{~K}$ required to produce ${ }^{56} \mathrm{Ni}$. Lower mass stars have steeper density gradients near the core. The largest ${ }^{56} \mathrm{Ni}$ mass should be $\sim 0.5 \mathrm{M}_{\odot}$ and should characterize 
the most energetic explosions (say a few times $10^{51} \mathrm{erg}$ ) in the most massive stars that explode by core bounce or neutrino energy transport $\left(25 \leqslant \mathrm{M} / \mathrm{M}_{\odot} \leqslant 70\right)$. In a $15 \mathrm{M}_{\odot}$ model we have studied, ${ }^{56} \mathrm{Ni}$ synthesis ranges from 0 to $0.1 \mathrm{M}_{\odot}$ depending upon the energy of the explosion. For explosion energies $\leqslant 5 \times 10^{50} \mathrm{erg}$, the inner nickel-rich layers may fall back onto the neutron star. For still lighter stars, especially 8 to $10 \mathrm{M}_{\odot}$, the ${ }^{56} \mathrm{Ni}$ synthesis is still less, perhaps a hundredth of a solar mass, though this has yet to be calculated. Of special interest will be the ${ }^{56} \mathrm{Ni}$ produced in the collapse of an accreting white dwarf (§3.2). Wilson and Mayle are working on that.

T. Ebisuzaki: I would guess that newly born neutron stars should evolve along the equivalent of the Hayashi track due to convection, I guess. What do you think about this idea?

Woosley: This is very much in line with Burrow's question above and the work he is doing on the early evolution of neutron stars. I think that you are right, i.e., that a surface convection zone of increasing depth will play an important role in removing the entropy of a young neutron star (or black hole). Such convection is also the essential aspect of Arnett's recently proposed model for boosting the neutrino flux during the explosion itself. Obvious implications loom for the surface magnetic field, configuration and strength in a young neutron star.

G. S. Bisnovatyi-Kogan: A remark about two important physical processes in supernova explosions. First, the interaction of neutrino flux from the core with falling envelope is very important and strongly reduces the falling velocity and bounce, as was shown by Nadëzhin in 1977. So the results of Hillebrandt et al. $[1982,1984]$ have not only been not confirmed later, but also earlier. Second, the magnetic field in rapidly rotating cores may transform the rotational energy into the energy of explosion and give SN explosion (magnetorotational) even when there is no explosion at the end of initial collapse. We are now working on this problem.

Woosley: Hillebrandt is not here to defend his work, but it is my understanding that his calculations include a very detailed and physical treatment of neutrino momentum and energy transport throughout the core implosion and explosion. The initial models that he starts with are provided by our group at a time when the central density has first reached $10^{10} \mathrm{~g} \mathrm{~cm}^{-3}$, thus neutrino momentum transport at an earlier stage is unlikely. I do not think that the braking of infalling matter by neutrinos is the cause for descrepancy with more recent calculations. Rather such differences are attribable to disparate treatments of nuclear equation of state, nuclear burning, neutrino energy transport in and behind the shock, and slight differences in the pre-explosive models (e.g. Hillebrandt et al. 1984; Wilson et al. 1986).

The possible importance of magnetic energy in obtaining a supernova explosion has stressed in a number of calculations (Le Blanc and Wilson 1970; Bisnovatyi-Kogan 1971, 1980; Bisnovatyi-Kogan et al. 1976; Meier et al. 1976; Müller and Hillebrandt 1979; Ohnishi 1983). In general, the results are quite sensitive to uncertain assumptions that are made, but show that large magnetic fields might be instrumental in obtaining explosions (though the works listed above 
come to differing conclusions in this regard). Unfortunately the calculations are some of the most difficult in all of astrophysics.

S. R. Kulkarni: You described two different mechanisms to get velocity kicks during collapse to a neutron star. Are both these mechanisms also operative in neutron stars formed by accreting white dwarfs going over the Chandrashekar mass limit? Observationally one may have to invoke kicks for neutron stars formed from massive stars to explain observed pulsar velocity distributions. [But] if the kick mechanism(s) is also operative in neutron stars born in low-mass systems then virtually none of the low-mass binaries (radio pulsars and X-ray binaries) can be formed.

Woosley: Yes, both mechanisms would operate to some extent in collapsing white dwarfs as well, especially the neutrino momentum asymmetry. But lacking quantitative estimates it is difficult to know how "robust" these kick mechanisms are. I envision them as stochastic. Thus large peculiar velocities may be obtained sometimes and smaller ones at others. It also makes a big difference whether the kick occurs along the orbital velocity vector or in opposition. As Burrows and I (1986) have described, a kick may sometimes be necessary to keep a binary bound.

S. Reynolds: Could you comment on the possible formation, in a rotating star, of a metastable core that takes a year or so to settle to a neutron star? Could you make more massive neutron stars that way?

Woosley: Maintaining a neutron star above its critical mass by supporting the mass, in part, with rotation is regarded as difficult. Tri-axial deformation would lead to gravitational radiation and angular momentum loss. The possibility of "fizzlers" (T. Gold) has been most recently reviewed by Kiguchi and Sato (1986) with negative conclusions. 